\title{
Complicated vaginal prolapses in two buffalo cows from the same ranch
}

Antônio Carlos Lopes Câmara, Igor Louzada Moreira, Anna Beatriz Veltri Peneiras, José Renato Junqueira Borges, Cristiane da Silva Pereira"

Hospital Escola de Grandes Animais, Faculdade de Agronomia e Medicina Veterinária, Universidade de Brasília(UnB), Brasília, DF, Brazil

*Corresponding author

e-mail: cristianepereira@unb.br

\section{Abstract}

Vaginal and cervical prolapses occur mostly in the last trimester of gestation, but may occasionally occur during estrus or the early postpartum period. Despite the greater worldwide incidence in buffaloes compared to cattle, the reports in Brazil are rare. Therefore, the present work aimed to report the two cases of complicated vaginal prolapses in buffalo cows from the same ranch. The rancher stated that each buffalo cow gave birth to a buffalo calf with no complications. The cows and offsprings were released on the paddock with the rest of the herd (about 80 buffaloes) and on the next day presented vaginal eversion. No treatment was performed and after 15 days the owner sought veterinary assistance. Gynecological evaluation was performed after mild sedation (xylazine: $0.025 \mathrm{mg} \mathrm{kg}^{-1}$, IV) and epidural anesthesia ( $5 \mathrm{~mL}$ of $2 \%$ lidocaine). Both buffalo cows presented severe cervicovaginal eversion. The more severely affected cow (Cow 1) was pluripara and about 8-years old, presenting extensive laceration of the vaginal mucosa with necrotic tissue, exposing the also necrotic urinary bladder wall. There was also prolapse of the rectum with extensive necrosis. The primipara cow (Cow 2) was about 3-years old and presented the entire vaginal mucosa and cervix protrude through the vulvar lips with entrapment of the urinary bladder. After proper cleaning and emptying the bladders by urethral catheterization, we tried to reposition both vaginal prolapses but the everted tissue presented severe fibrosis and large amounts of necrotic tissues, making impossible do replace it. Due to the cost benefit analysis and reserved prognosis, the owner opted to slaughter the cows. Etiologic factors of vaginal prolapse in buffaloes may be attributed to nutritional imbalance, hormonal imbalance, seasonal-management, and hereditary predisposition. Since both buffalo cows are from the same ranch, nutritional imbalance was better investigated and corrected. Recent studies classifies vaginal prolapses into 4 grades, according to the severity of eversion and the extent of injury. According to this, Cow 1 and 2 
presented grade $4 \mathrm{~b}$ and grade 3 vaginal prolapses, respectively. Grade $4 \mathrm{~b}$ eversion is considered the most severe form in which the cervicovaginal eversion persists for such a duration that the entire vaginal mucosa appears necrotic and fibrotic. Infection may become so extensive that the urinary bladder becomes necrotic and septic peritonitis may ensue. The delay by the rancher in seeking specialized veterinary care severely worsened the prognosis of the disease in these cases. Pre or pospartum vaginal or cervical prolapses should be replaced and secured promptly before contamination, laceration, sepsis, fibrosis, and necrosis occur. Vaginal eversion must be consider a reproductive emergency and producers should also be educated to seek veterinary assistance as fast as possible. 